\title{
Uniqueness and Existence of Solution for a System of Fractional $q$-Difference Equations
}

\author{
Wen-Xue Zhou ${ }^{1,2}$ and Hai-zhong Liu ${ }^{1}$ \\ ${ }^{1}$ College of Mathematics and Physics, Lanzhou Jiaotong University, Lanzhou 730070, China \\ ${ }^{2}$ School of Mathematical Sciences, Fudan University, Shanghai 200433, China \\ Correspondence should be addressed to Wen-Xue Zhou; wxzhou2006@126.com
}

Received 28 March 2014; Revised 27 May 2014; Accepted 13 June 2014; Published 7 July 2014

Academic Editor: Felix Sadyrbaev

Copyright (c) 2014 W.-X. Zhou and H.-z. Liu. This is an open access article distributed under the Creative Commons Attribution License, which permits unrestricted use, distribution, and reproduction in any medium, provided the original work is properly cited.

We prove the existence and uniqueness of solution for a system of fractional differential equations. Our results are based on the nonlinear alternative of Leray-Schauder type and Banach's fixed-point theorem.

\section{Introduction}

This paper is mainly concerned with the uniqueness and existence of solution for a system of fractional $q$-difference equations given by

$$
\begin{gathered}
{ }^{C} D_{q}^{\alpha} u(t)=f(t, v(t)), \quad 1<\alpha \leq 2, t \in[0,1], \\
{ }^{C} D_{q}^{\beta} v(t)=g(t, u(t)), \quad 1<\beta \leq 2, t \in[0,1], \\
\alpha_{1} u(0)-\beta_{1} D_{q} u(0)=\gamma_{1} u\left(\eta_{1}\right), \\
\alpha_{2} u(1)+\beta_{2} D_{q} u(1)=\gamma_{2} u\left(\eta_{2}\right), \\
\alpha_{3} v(0)-\beta_{3} D_{q} v(0)=\gamma_{3} v\left(\eta_{3}\right), \\
\alpha_{4} v(1)+\beta_{4} D_{q} v(1)=\gamma_{4} v\left(\eta_{4}\right),
\end{gathered}
$$

where ${ }^{C} D_{q}^{\alpha},{ }^{C} D_{q}^{\beta}$ is the fractional $q$-derivatives of the Caputo type, $1<\alpha, \beta \leq 2, \alpha_{i}(i=1,2,3,4), \beta_{i}(i=1,2,3,4), \gamma_{i}(i=$ $1,2,3,4)$, and $\eta_{i}(i=1,2,3,4)$ are arbitrary real constants, and $f, g:[0,1] \times \mathbb{R} \rightarrow \mathbb{R}$ are given continuous functions.

In the last few years, fractional differential equations (in short FDEs) have been studied extensively. The motivation for those works stems from both the development of the theory of fractional calculus itself and the applications of such constructions in various sciences such as physics, mechanics, chemistry, and engineering. For an extensive collection of such results, we refer the readers to the monographs by Kilbas et al. [1], Miller and Ross [2], Oldham and Spanier [3], Podlubny [4], and Samko et al. [5].

Some basic theory for the initial value problems of fractional differential equations involving Riemann-Liouville differential operator has been discussed by Lakshmikantham and Vatsala ([6-8]), Babakhani and Daftardar-Gejji ([9-11]), Bai [12], and so on. Also, there are some papers which deal with the existence and multiplicity of solutions (or positive solution) for nonlinear FDE of BVPs by using techniques of nonlinear analysis (fixed-point theorems, Leray-Shauder theory, topological degree theory, etc.) - see ([13-18]) and the references therein. The study of a coupled system of fractional order is also very significant because this kind of system can often occur in applications. The reader is referred to the papers ([19-22]) and the references cited therein.

The pioneer work on $q$-difference calculus or quantum calculus dates back to Jackson's papers $([23,24])$, while a systematic treatment of the subject can be found in $[25,26]$. For some recent existence results on $q$-difference equations, see [27-29] and the references therein.

There has also been a growing interest on the subject of discrete fractional equations on the time scale $\mathbb{Z}$. Some interesting results on the topic can be found in a series of papers [30-38]. Fractional $q$-difference equations have recently attracted the attention of several researchers. For some earlier work on the topic, we refer to $[39,40]$, whereas some recent work on the existence theory of fractional 
$q$-difference equations can be found in [41-45]. However, the study of boundary value problems of fractional $q$-difference equations is at its infancy and much of the work on the topic is yet to be done.

From the above works, we can see a fact, although the fractional boundary value problems have been investigated by some authors. To the best of our knowledge, there have been few papers which deal with problem (1) for nonlinear fractional differential equation. Motivated by all the works above, in this paper we discuss problem (1). Using nonlinear alternative of Leray-Schauder type, we will give the existence and uniqueness of solution for a system of fractional differential equations with Riemann-Liouville integral boundary conditions of different order (1).

The paper is organized as follows. In Section 2, we give some preliminary results that will be used in the proof of the main results. In Section 3, we establish the uniqueness and existence of a solution for the nonlinear fractional differential equation boundary value problem (1). In last section, we give two examples to illustrate our work.

\section{Preliminaries and Lemmas}

In this section, we cite some definitions and fundamental results of the $q$-calculus as well as of the fractional $q$-calculus $([46,47])$. We also give a lemma that will be used in obtaining the main results of the paper.

Let $q \in(0,1)$ and define [47]

$$
[a]_{q}=\frac{q^{a}-1}{q-1}=a^{a-1}+\cdots+1, \quad a \in \mathbb{R} .
$$

The $q$-analogue of the power $(a-b)^{n}$ is

$$
\begin{gathered}
(a-b)^{(0)}=1, \\
(a-b)^{(n)}=\prod_{k=0}^{n-1}\left(a-b q^{k}\right), \quad a, b \in \mathbb{R}, n \in \mathbb{N} .
\end{gathered}
$$

If $\alpha$ is not a positive integer, then

$$
(a-b)^{(\alpha)}=a^{\alpha} \prod_{i=0}^{\infty} \frac{\left(1-(b / a) q^{i}\right)}{\left(1-(b / a) q^{\alpha+i}\right)} .
$$

Note that if $b=0$, then $a^{(\alpha)}=a^{\alpha}$. The $q$-gamma function is defined by

$$
\Gamma_{q}(x)=\frac{(1-q)^{(x-1)}}{(1-q)^{x-1}}, \quad x \in \mathbb{R} \backslash\{0,-1,-2, \ldots\}, 0<q<1
$$

and satisfies $\Gamma_{q}(x+1)=[x]_{q} \Gamma_{q}(x)$ (see [47]).

The $q$-derivative of a function $f$ is here defined by

$$
D_{q} f(x)=\frac{d_{q} f(x)}{d_{q} x}=\frac{f(q x)-f(x)}{(q-1) x}
$$

and $q$-derivatives of higher order by

$$
D_{q}^{n} f(x)= \begin{cases}f(x), & \text { if } n=0 \\ D_{q} D_{q}^{n-1} f(x), & \text { if } n \in \mathbb{N}\end{cases}
$$

The $q$-integral of a function $f$ defined in the interval $[0, b]$ is given by

$$
\begin{array}{r}
\int_{0}^{x} f(t) d_{q} t=x(1-q) \sum_{n=0}^{\infty} f\left(x q^{n}\right) q^{n}, \\
0 \leq|q|<1, \quad x \in[0, b] .
\end{array}
$$

If $a \in[0, b]$ and $f$ is defined in the interval $[0, b]$, its integral from $a$ to $b$ is defined by

$$
\int_{a}^{b} f(t) d_{q} t=\int_{0}^{b} f(t) d_{q} t-\int_{0}^{a} f(t) d_{q} t
$$

Similarly, as done for derivatives, an operator $I_{q}^{n}$ can be defined, namely, by

$$
\begin{gathered}
\left(I_{q}^{0} f\right)(x)=f(x), \\
\left(I_{q}^{n} f\right)(x)=I_{q}\left(I_{q}^{n-1} f\right)(x), \quad n \in \mathbb{N} .
\end{gathered}
$$

The fundamental theorem of calculus applies to these operators $I_{q}$ and $D_{q}$; that is,

$$
\left(D_{q} I_{q} f\right)(x)=f(x)
$$

and if $f$ is continuous at $x=0$, then

$$
\left(I_{q} D_{q} f\right)(x)=f(x)-f(0) .
$$

Basic properties of the two operators can be found in the book that is mentioned in [8]. We now point out three formulas that will be used later $\left({ }_{i} D_{q}\right.$ denotes the derivative with respect to variable $i)$ [43]:

$$
\begin{gathered}
{[a(t-s)]^{(\alpha)}=a^{\alpha}(t-s)^{(\alpha)},} \\
{ }_{t} D_{q}(t-s)^{(\alpha)}=[\alpha]_{q}(t-s)^{(\alpha-1)}, \\
\left({ }_{x} D_{q} \int_{0}^{x} f(x, t) d_{q} t\right)(x)=\int_{0}^{x}{ }_{x} D_{q} f(x, t) d_{q} t+f(q x, x) .
\end{gathered}
$$

Remark 1. We note that if $\alpha>0$ and $a \leq b \leq t$, then $(t-a)^{(\alpha)} \geq(t-b)^{(\alpha)}[43]$.

Definition 2 (see [40]). Let $\alpha \geq 0$ and let $f$ be a function defined on $[0,1]$. The fractional $q$-integral of the RiemannLiouville type is $\left({ }_{\mathrm{RL}} I_{q}^{0} f\right)(x)=f(x)$ and

$$
\begin{array}{r}
\left({ }_{\mathrm{RL}} I_{q}^{\alpha} f\right)(x)=\int_{0}^{x} \frac{(x-q t)^{(\alpha-1)}}{\Gamma_{q}(\alpha)} f(t) d_{q} t, \\
\alpha \in \mathbb{R}^{+}, x \in[0,1] .
\end{array}
$$


Definition 3 (see [48]). The fractional $q$-derivative of the Riemann-Liouville type of order $\alpha \geq 0$ is defined by $\left({ }_{\mathrm{RL}} D_{q}^{0} f\right)(x)=f(x)$ and

$$
\left({ }_{\mathrm{RL}} D_{q}^{\alpha} f\right)(x)=\left(D_{q}^{[\alpha]} I_{q}^{[\alpha]-\alpha} f\right)(x), \quad \alpha>0,
$$

where $[\alpha]$ is the smallest integer greater than or equal to $\alpha$.

Definition 4 (see [48]). The fractional $q$-derivative of the Caputo type of order $\alpha \geq 0$ is defined by

$$
\left({ }^{C} D_{q}^{\alpha} f\right)(x)=\left(I_{q}^{[\alpha]-\alpha} D_{q}^{[\alpha]} f\right)(x), \quad \alpha>0,
$$

where $[\alpha]$ is the smallest integer greater than or equal to $\alpha$.

Lemma 5. Let $\alpha, \beta \geq 0$ and let $f$ be a function defined on $[0,1]$. Then the next formulas hold:

(1) $\left(I_{q}^{\beta} I_{q}^{\alpha} f\right)(x)=\left(I_{q}^{\alpha+\beta} f\right)(x)$,

(2) $\left(D_{q}^{\alpha} I_{q}^{\alpha} f\right)(x)=f(x)$.

Lemma 6 (see [42]). Let $\alpha \geq 0$ and $n \in \mathbb{N}$. Then the following equality holds:

$$
\begin{aligned}
\left({ }_{R L} I_{q R L}^{\alpha} D_{q}^{n} f\right)(x)= & { }_{R L} D_{q R L}^{n} I_{q}^{\alpha} f(x) \\
& -\sum_{k=0}^{\alpha-1} \frac{x^{\alpha-n+k}}{\Gamma_{q}(\alpha+k-n+1)}\left(D_{q}^{k} f\right)(0) .
\end{aligned}
$$

Lemma 7 (see [48]). Let $\alpha>0$ and $n \in \mathbb{R}^{+} \backslash \mathbb{N}$. Then the following equality holds:

$$
\left(I_{q}^{\alpha C} D_{q}^{\alpha} f\right)(x)=f(x)-\sum_{k=0}^{[\alpha]-1} \frac{x^{k}}{\Gamma_{q}(k+1)}\left(D_{q}^{k} f\right)(0) .
$$

For convenience, one introduces the following notations:

$$
\begin{gathered}
b_{1}=\frac{\gamma_{1}\left(\alpha_{2}-\gamma_{2}\right)}{\Delta}, \quad b_{2}=\frac{\gamma_{1}\left(\alpha_{2}+\beta_{2}-\gamma_{2} \eta_{2}\right)}{\Delta}, \\
b_{3}=\frac{\left(\alpha_{1}-\gamma_{1}\right)}{\Delta}, \quad b_{4}=\frac{\left(\beta_{1}+\gamma_{1} \eta_{1}\right)}{\Delta}, \\
\Delta=\left(\gamma_{2}-\alpha_{2}\right)\left(\beta_{1}+\gamma_{1} \eta_{1}\right)+\left(\gamma_{1}-\alpha_{1}\right)\left(\alpha_{2}+\beta_{2}-\gamma_{2} \eta_{2}\right) .
\end{gathered}
$$

From Lemmas 5 and 7, we can obtain the following lemma.

Lemma 8. Let $h \in C[0,1]$ and $\Delta \neq 0$; then the unique solution of the linear fractional boundary value problem

$$
\begin{gathered}
{ }^{C} D_{q}^{\alpha} u(t)=h(t), \quad 1<\alpha \leq 2, t \in[0,1], \\
\alpha_{1} u(0)-\beta_{1} D_{q} u(0)=\gamma_{1} u\left(\eta_{1}\right), \\
\alpha_{2} u(1)+\beta_{2} D_{q} u(1)=\gamma_{2} u\left(\eta_{2}\right)
\end{gathered}
$$

is given by

$$
\begin{aligned}
u(t)= & \int_{0}^{t} \frac{(t-q s)^{(\alpha-1)}}{\Gamma_{q}(\alpha)} h(s) d_{q} s \\
& +\left(b_{1} t+b_{2}\right) \int_{0}^{\eta_{1}} \frac{\left(\eta_{1}-q s\right)^{(\alpha-1)}}{\Gamma_{q}(\alpha)} h(s) d_{q} s \\
& +\left(b_{3} t+b_{4}\right)\left[-\gamma_{2} \int_{0}^{\eta_{2}} \frac{\left(\eta_{2}-q s\right)^{(\alpha-1)}}{\Gamma_{q}(\alpha)} h(s) d_{q} s\right. \\
& +\alpha_{2} \int_{0}^{1} \frac{(1-q s)^{(\alpha-1)}}{\Gamma_{q}(\alpha)} h(s) d_{q} s \\
& \left.+\beta_{2} \int_{0}^{1} \frac{(1-q s)^{(\alpha-2)}}{\Gamma_{q}(\alpha-1)} h(s) d_{q} s\right] .
\end{aligned}
$$

The following lemma is fundamental in the proofs of our main result.

Lemma 9 (see [49]; nonlinear alternative of Leray-Schauder type). Let $E$ be a Banach space with $M \subseteq E$ closed and convex. Assume that $U$ is a relatively open subset of $C$ with $0 \in U$ and $F: \bar{U} \rightarrow C$ is continuous, compact (i.e., $F(U)$ is a relatively compact subset of C) map. Then either

(i) F has a fixed point in $\bar{U}$ or

(ii) there exist $u \in \partial U$ and $\lambda \in(0,1)$ with $u=\lambda F u$.

\section{Main Results}

In this section, we will discuss the uniqueness and existence of solutions for boundary value problem (1).

First of all, we define the Banach space $X=\{u \mid u \in$ $C[0,1]\}$ endowed with the norm $\|u\|=\max _{t \in[0,1]}|u(t)|$. For $(u, v) \in X \times X$, let $\|(u, v)\|=\max \{\|u\|,\|v\|\}$; then $(X \times$ $X,\|(\cdot, \cdot)\|)$ is a Banach space.

For convenience, we set

$$
\boldsymbol{\Delta}=\left(\gamma_{4}-\alpha_{4}\right)\left(\beta_{3}+\gamma_{3} \eta_{3}\right)+\left(\gamma_{3}-\alpha_{3}\right)\left(\alpha_{4}+\beta_{4}-\gamma_{4} \eta_{4}\right) \text {, }
$$

and let $\boldsymbol{\Delta} \neq 0$. Note

$$
\begin{array}{cc}
\mathfrak{b}_{1}=\frac{\gamma_{3}\left(\alpha_{4}-\gamma_{4}\right)}{\Delta}, & \mathfrak{b}_{2}=\frac{\gamma_{3}\left(\alpha_{4}+\beta_{4}-\gamma_{4} \eta_{4}\right)}{\Delta}, \\
\mathfrak{b}_{3}=\frac{\left(\alpha_{3}-\gamma_{3}\right)}{\boldsymbol{\Delta}}, & \mathfrak{b}_{4}=\frac{\left(\beta_{3}+\gamma_{3} \eta_{3}\right)}{\boldsymbol{\Delta}} .
\end{array}
$$

Employing Lemma 8, system (1) can be expressed as

$$
\begin{aligned}
u(t)= & \int_{0}^{t} \frac{(t-q s)^{(\alpha-1)}}{\Gamma_{q}(\alpha)} f(s, v(s)) d_{q} s \\
& +\left(b_{1} t+b_{2}\right) \int_{0}^{\eta_{1}} \frac{\left(\eta_{1}-q s\right)^{(\alpha-1)}}{\Gamma_{q}(\alpha)} f(s, v(s)) d_{q} s
\end{aligned}
$$




$$
\begin{gathered}
+\left(b_{3} t+b_{4}\right)\left[-\gamma_{2} \int_{0}^{\eta_{2}} \frac{\left(\eta_{2}-q s\right)^{(\alpha-1)}}{\Gamma_{q}(\alpha)} f(s, v(s)) d_{q} s\right. \\
+\alpha_{2} \int_{0}^{1} \frac{(1-q s)^{(\alpha-1)}}{\Gamma_{q}(\alpha)} f(s, v(s)) d_{q} s \\
\left.+\beta_{2} \int_{0}^{1} \frac{(1-q s)^{(\alpha-2)}}{\Gamma_{q}(\alpha-1)} f(s, v(s)) d_{q} s\right], \\
v(t)=\int_{0}^{t} \frac{(t-q s)^{(\beta-1)}}{\Gamma_{q}(\beta)} g(s, u(s)) d_{q} s \\
\left.+\left(\mathfrak{b}_{1} t+\mathfrak{b}_{2}\right) \int_{0}^{\eta_{3}} \frac{\left(\eta_{3}-q s\right)^{(\beta-1)}}{\Gamma_{q}(\beta)} u(s)\right) d_{q} s \\
+\left(\mathfrak{b}_{3} t+\mathfrak{b}_{4}\right)\left[-\gamma_{4} \int_{0}^{\eta_{4}} \frac{\left(\eta_{4}-q s\right)^{(\beta-1)}}{\Gamma_{q}(\beta)} g(s, u(s)) d_{q} s\right. \\
+\beta_{4} \int_{0}^{1} \frac{(1-q s)^{(\beta-1)}}{\Gamma_{q}(\beta)} g(s, u(s)) d_{q} s \\
\Gamma_{q}(\beta-1) \\
\left.+(s, u(s)) d_{q} s\right],
\end{gathered}
$$

where $b_{1}, b_{2}, b_{3}, b_{4}$ are given by (21), and $\mathfrak{b}_{1}, \mathfrak{b}_{2}, \mathfrak{b}_{3}, \mathfrak{b}_{4}$ are given by (22)

From Lemma 8 in Section 2, we can obtain the following lemma.

Lemma 10. Suppose that $f(t, v)$ and $g(t, u)$ are continuous; then $(u, v) \in X \times X$ is a solution of $B V P(1)$ if and only if $(u, v) \in$ $X \times X$ is a solution of the integral equations (24).

Let $(u, v) \in X \times X$; define an operator $T: X \times X \rightarrow X \times X$ as

$$
T(u, v)(t)=\left(T_{1} v(t), T_{2} u(t)\right)
$$

where

$$
\begin{aligned}
& T_{1} v(t) \\
& =\int_{0}^{t} \frac{(t-q s)^{(\alpha-1)}}{\Gamma_{q}(\alpha)} f(s, v(s)) d_{q} s \\
& +\left(b_{1} t+b_{2}\right) \int_{0}^{\eta_{1}} \frac{\left(\eta_{1}-q s\right)^{(\alpha-1)}}{\Gamma_{q}(\alpha)} f(s, v(s)) d_{q} s \\
& +\left(b_{3} t+b_{4}\right)\left[-\gamma_{2} \int_{0}^{\eta_{2}} \frac{\left(\eta_{2}-q s\right)^{(\alpha-1)}}{\Gamma_{q}(\alpha)} f(s, v(s)) d_{q} s\right. \\
& +\alpha_{2} \int_{0}^{1} \frac{(1-q s)^{(\alpha-1)}}{\Gamma_{q}(\alpha)} f(s, v(s)) d_{q} s \\
& \left.+\beta_{2} \int_{0}^{1} \frac{(1-q s)^{(\alpha-2)}}{\Gamma_{q}(\alpha-1)} f(s, v(s)) d_{q} s\right]
\end{aligned}
$$

$T_{2} u(t)$

$$
\begin{aligned}
& =\int_{0}^{t} \frac{(t-q s)^{(\beta-1)}}{\Gamma_{q}(\beta)} g(s, u(s)) d_{q} s \\
& +\left(\mathfrak{b}_{1} t+\mathfrak{b}_{2}\right) \int_{0}^{\eta_{3}} \frac{\left(\eta_{3}-q s\right)^{(\beta-1)}}{\Gamma_{q}(\beta)} g(s, u(s)) d_{q} s \\
& +\left(\mathfrak{b}_{3} t+\mathfrak{b}_{4}\right)\left[-\gamma_{4} \int_{0}^{\eta_{4}} \frac{\left(\eta_{4}-q s\right)^{(\beta-1)}}{\Gamma_{q}(\beta)} g(s, u(s)) d_{q} s\right. \\
& +\alpha_{4} \int_{0}^{1} \frac{(1-q s)^{(\beta-1)}}{\Gamma_{q}(\beta)} g(s, u(s)) d_{q} s \\
& \left.+\beta_{4} \int_{0}^{1} \frac{(1-q s)^{(\beta-2)}}{\Gamma_{q}(\beta-1)} g(s, u(s)) d_{q} s\right]
\end{aligned}
$$

then, by Lemma 10, the fixed point of operator $T$ coincides with the solution of system (1).

In the first result, we prove uniqueness of solution of the boundary value problem (1) via Banach's contraction principle.

Theorem 11. Assume that $f, g:[0,1] \times \mathbb{R} \rightarrow \mathbb{R}$ are continuous functions and the following conditions hold:

$(\mathrm{H} 1)$ there exist two q-integrable functions $L_{1}, L_{2}:[0,1] \rightarrow$ $\mathbb{R}$ that satisfy

$$
\begin{aligned}
& |f(t, u)-f(t, v)| \leq L_{1}(t)|u-v|, \quad \forall t \in[0,1], u, v \in \mathbb{R} ; \\
& |g(t, u)-g(t, v)| \leq L_{2}(t)|u-v|, \quad \forall t \in[0,1], u, v \in \mathbb{R} .
\end{aligned}
$$

In addition, assume that

$$
\begin{aligned}
\kappa_{1}:= & \left(1+\left|\alpha_{2}\right| \delta_{2}\left(I_{q}^{\alpha} L_{1}\right)(1)+\left|\gamma_{1}\right| \delta_{1}\left(I_{q}^{\alpha} L_{1}\right)\left(\eta_{1}\right)\right) \\
& +\left|\gamma_{2}\right| \delta_{2}\left(I_{q}^{\alpha} L_{1}\right)\left(\eta_{2}\right)+\left|\beta_{2}\right| \delta_{2}\left(I_{q}^{\alpha-1} L_{1}\right)(1)<1, \\
\kappa_{2}:= & \left(1+\left|\alpha_{4}\right| \delta_{4}\left(I_{q}^{\beta} L_{2}\right)(1)+\left|\gamma_{3}\right| \delta_{3}\left(I_{q}^{\beta} L_{2}\right)\left(\eta_{3}\right)\right) \\
& +\left|\gamma_{4}\right| \delta_{4}\left(I_{q}^{\beta} L_{2}\right)\left(\eta_{4}\right)+\left|\beta_{4}\right| \delta_{4}\left(I_{q}^{\beta-1} L_{2}\right)(1)<1,
\end{aligned}
$$

where

$$
\begin{aligned}
& \delta_{1}:=\frac{\left|\alpha_{2}-\gamma_{2}\right|+\left|\alpha_{2}+\beta_{2}-\gamma_{2} \eta_{2}\right|}{|\Delta|}, \\
& \delta_{2}:=\frac{\left|\alpha_{1}-\gamma_{1}\right|+\left|\beta_{1}-\gamma_{1} \eta_{1}\right|}{|\Delta|}, \\
& \delta_{3}:=\frac{\left|\alpha_{4}-\gamma_{4}\right|+\left|\alpha_{4}+\beta_{4}-\gamma_{4} \eta_{4}\right|}{|\mathbf{\Delta}|}, \\
& \delta_{4}:=\frac{\left|\alpha_{3}-\gamma_{3}\right|+\left|\beta_{3}-\gamma_{3} \eta_{3}\right|}{|\mathbf{\Delta}|},
\end{aligned}
$$

where $\Delta$ and $\Delta$ are given by (19) and (22), respectively. Then system (1) has a unique solution. 
Proof. Let us set $\sup _{t \in[0,1]}|f(t, 0)|=M_{1}<\infty, \sup _{t \in[0,1]}$ $|g(t, 0)|=M_{2}<\infty$,

$$
\begin{aligned}
A_{1}= & \frac{1}{\Gamma_{q}(\alpha+1)}\left(1+\left|\gamma_{1}\right| \delta_{1} \eta_{1}^{(\alpha-1)}+\left|\gamma_{2}\right| \delta_{2} \eta_{2}^{(\alpha-1)}+\left|\alpha_{2}\right| \delta_{2}\right) \\
& +\frac{\left|\beta_{2}\right| \delta_{2}}{\Gamma_{q}(\alpha)}, \\
A_{2}= & \frac{1}{\Gamma_{q}(\beta+1)}\left(1+\left|\gamma_{3}\right| \delta_{3} \eta_{3}^{(\beta-1)}+\left|\gamma_{4}\right| \delta_{4} \eta_{4}^{(\beta-1)}+\left|\alpha_{4}\right| \delta_{4}\right) \\
& +\frac{\left|\beta_{4}\right| \delta_{4}}{\Gamma_{q}(\beta)} .
\end{aligned}
$$

Define

$$
\begin{gathered}
U=\{(u, v) \in X \times X:\|(u, v)\| \leq r\}, \\
B_{r_{1}}=\left\{u \in C[0,1]:|u| \leq r_{1}\right\}, \\
B_{r_{2}}=\left\{v \in C[0,1]:|v| \leq r_{2}\right\} .
\end{gathered}
$$

For $v \in B_{r_{1}}$, we obtain

$$
\begin{aligned}
|f(t, v(t))| & \leq|f(t, v(t))-f(t, 0)|+|f(t, 0)| \\
& \leq L_{1}(t)|v(t)|+|f(t, 0)| \\
& \leq L_{1}(t) r_{1}+M_{1} .
\end{aligned}
$$

Then, for $v \in B_{r_{1}}, t \in[0,1]$, we have

$\left\|T_{1} v\right\|$

$$
\begin{aligned}
\leq \sup _{t \in[0,1]}\{ & \int_{0}^{t} \frac{(t-q s)^{(\alpha-1)}}{\Gamma_{q}(\alpha)}|f(s, v(s))| d_{q} s \\
& +\left|\frac{\gamma_{1}}{\Delta}\left[\left(\alpha_{2}-\gamma_{2}\right) t-\left(\alpha_{2}+\beta_{2}-\gamma_{2} \eta_{2}\right)\right]\right| \\
& \times \int_{0}^{\eta_{1}} \frac{\left(\eta_{1}-q s\right)^{(\alpha-1)}}{\Gamma_{q}(\alpha)}|f(s, v(s))| d_{q} s \\
& +\left|\frac{\gamma_{2}}{\Delta}\left[\left(\alpha_{1}-\gamma_{1}\right) t+\left(\beta_{1}+\gamma_{1} \eta_{1}\right)\right]\right| \\
& \times \int_{0}^{\eta_{2}} \frac{\left(\eta_{2}-q s\right)^{(\alpha-1)}}{\Gamma_{q}(\alpha)}|f(s, v(s))| d_{q} s \\
& +\left|\frac{\alpha_{2}}{\Delta}\left[\left(\alpha_{1}-\gamma_{1}\right) t+\left(\beta_{1}+\gamma_{1} \eta_{1}\right)\right]\right| \\
& \times \int_{0}^{1} \frac{(1-q s)^{(\alpha-1)}}{\Gamma_{q}(\alpha)}|f(s, v(s))| d_{q} s \\
& +\left|\frac{\beta_{2}}{\Delta}\left[\left(\alpha_{1}-\gamma_{1}\right) t+\left(\beta_{1}+\gamma_{1} \eta_{1}\right)\right]\right| \\
& \left.\times \int_{0}^{1} \frac{(1-q s)^{(\alpha-2)}}{\Gamma_{q}(\alpha-1)}|f(s, v(s))| d_{q} s\right\}
\end{aligned}
$$

$$
\begin{aligned}
& \leq \sup _{t \in[0,1]}\left\{\int_{0}^{t} \frac{(t-q s)^{(\alpha-1)}}{\Gamma_{q}(\alpha)}\left[L_{1}(s) r_{1}+M_{1}\right] d_{q} s\right. \\
& +\left|\frac{\gamma_{1}}{\Delta}\left[\left(\alpha_{2}-\gamma_{2}\right) t-\left(\alpha_{2}+\beta_{2}-\gamma_{2} \eta_{2}\right)\right]\right| \\
& \times \int_{0}^{\eta_{1}} \frac{\left(\eta_{1}-q s\right)^{(\alpha-1)}}{\Gamma_{q}(\alpha)}\left[L_{1}(s) r_{1}+M_{1}\right] d_{q} s \\
& +\left|\frac{\gamma_{2}}{\Delta}\left[\left(\alpha_{1}-\gamma_{1}\right) t+\left(\beta_{1}+\gamma_{1} \eta_{1}\right)\right]\right| \\
& \times \int_{0}^{\eta_{2}} \frac{\left(\eta_{2}-q s\right)^{(\alpha-1)}}{\Gamma_{q}(\alpha)}\left[L_{1}(s) r_{1}+M_{1}\right] d_{q} s \\
& +\left|\frac{\alpha_{2}}{\Delta}\left[\left(\alpha_{1}-\gamma_{1}\right) t+\left(\beta_{1}+\gamma_{1} \eta_{1}\right)\right]\right| \\
& \times \int_{0}^{1} \frac{(1-q s)^{(\alpha-1)}}{\Gamma_{q}(\alpha)}\left[L_{1}(s) r_{1}+M_{1}\right] d_{q} s \\
& +\left|\frac{\beta_{2}}{\Delta}\left[\left(\alpha_{1}-\gamma_{1}\right) t+\left(\beta_{1}+\gamma_{1} \eta_{1}\right)\right]\right| \\
& \left.\times \int_{0}^{1} \frac{(1-q s)^{(\alpha-2)}}{\Gamma_{q}(\alpha-1)}\left[L_{1}(s) r_{1}+M_{1}\right] d_{q} s\right\} \\
& \leq M_{1}\left\{\int_{0}^{1} \frac{(1-q s)^{(\alpha-1)}}{\Gamma_{q}(\alpha)} d_{q} s+\left|\gamma_{1}\right| \delta_{1} \int_{0}^{\eta_{1}} \frac{\left(\eta_{1}-q s\right)^{(\alpha-1)}}{\Gamma_{q}(\alpha)} d_{q} s\right. \\
& +\left|\gamma_{2}\right| \delta_{2} \int_{0}^{\eta_{2}} \frac{\left(\eta_{2}-q s\right)^{(\alpha-1)}}{\Gamma_{q}(\alpha)} d_{q} s \\
& +\left|\gamma_{2}\right| \delta_{2} \int_{0}^{1} \frac{(1-q s)^{(\alpha-1)}}{\Gamma_{q}(\alpha)} d_{q} s \\
& \left.+\left|\gamma_{2}\right| \delta_{2} \int_{0}^{1} \frac{(1-q s)^{(\alpha-2)}}{\Gamma_{q}(\alpha-1)} d_{q} s\right\} \\
& +r_{1}\left\{\int_{0}^{1} \frac{(1-q s)^{(\alpha-1)}}{\Gamma_{q}(\alpha)} L_{1}(s) d_{q} s\right. \\
& +\left|\gamma_{1}\right| \delta_{1} \int_{0}^{\eta_{1}} \frac{\left(\eta_{1}-q s\right)^{(\alpha-1)}}{\Gamma_{q}(\alpha)} L_{1}(s) d_{q} s \\
& +\left|\gamma_{2}\right| \delta_{2} \int_{0}^{\eta_{2}} \frac{\left(\eta_{2}-q s\right)^{(\alpha-1)}}{\Gamma_{q}(\alpha)} L_{1}(s) d_{q} s \\
& +\left|\gamma_{2}\right| \delta_{2} \int_{0}^{1} \frac{(1-q s)^{(\alpha-1)}}{\Gamma_{q}(\alpha)} L_{1}(s) d_{q} s \\
& \left.+\left|\gamma_{2}\right| \delta_{2} \int_{0}^{1} \frac{(1-q s)^{(\alpha-2)}}{\Gamma_{q}(\alpha-1)} L_{1}(s) d_{q} s\right\}
\end{aligned}
$$




$$
\begin{aligned}
\leq M_{1}\{ & \frac{1}{\Gamma_{q}(\alpha+1)} \\
& \times\left(1+\left|\gamma_{1}\right| \delta_{1} \eta_{1}^{(\alpha-1)}+\left|\gamma_{2}\right| \delta_{2} \eta_{2}^{(\alpha-1)}+\left|\alpha_{2}\right| \delta_{2}\right) \\
& \left.+\frac{\left|\beta_{2}\right| \delta_{2}}{\Gamma_{q}(\alpha)}\right\} \\
+r_{1} & \left\{\int_{0}^{1} \frac{(1-q s)^{(\alpha-1)}}{\Gamma_{q}(\alpha)} L_{1}(s) d_{q} s\right. \\
& +\left|\gamma_{1}\right| \delta_{1} \int_{0}^{\eta_{1}} \frac{\left(\eta_{1}-q s\right)^{(\alpha-1)}}{\Gamma_{q}(\alpha)} L_{1}(s) d_{q} s \\
& +\left|\gamma_{2}\right| \delta_{2} \int_{0}^{\eta_{2}} \frac{\left(\eta_{2}-q s\right)^{(\alpha-1)}}{\Gamma_{q}(\alpha)} L_{1}(s) d_{q} s \\
& +\left|\alpha_{2}\right| \delta_{2} \int_{0}^{1} \frac{(1-q s)^{(\alpha-1)}}{\Gamma_{q}(\alpha)} L_{1}(s) d_{q} s \\
& \left.+\left|\beta_{2}\right| \delta_{2} \int_{0}^{1} \frac{(1-q s)^{(\alpha-2)}}{\Gamma_{q}(\alpha-1)} L_{1}(s) d_{q} s\right\}
\end{aligned}
$$

In view of (31), we obtain

$$
\left\|T_{1} v\right\| \leq M_{1} A_{1}+r_{1} \kappa_{1} .
$$

From the last estimate we deduce that $r_{1}=M_{1} A_{1} /\left(1-\kappa_{1}\right)$.

By a similar way as done above we have

$\left\|T_{2} u\right\|$

$$
\begin{aligned}
& \leq M_{2}\{ \frac{1}{\Gamma_{q}(\beta+1)} \\
& \times\left(1+\left|\gamma_{3}\right| \delta_{3} \eta_{3}^{(\beta-1)}+\left|\gamma_{4}\right| \delta_{4} \eta_{4}^{(\beta-1)}+\left|\alpha_{4}\right| \delta_{4}\right) \\
&\left.+\frac{\left|\beta_{4}\right| \delta_{4}}{\Gamma_{q}(\beta)}\right\} \\
&+r_{2}\left\{\int_{0}^{1} \frac{(1-q s)^{(\beta-1)}}{\Gamma_{q}(\beta)} L_{2}(s) d_{q} s\right. \\
&+\left|\gamma_{3}\right| \delta_{3} \int_{0}^{\eta_{3}} \frac{\left(\eta_{3}-q s\right)^{(\beta-1)}}{\Gamma_{q}(\beta)} L_{2}(s) d_{q} s \\
&+\left|\gamma_{4}\right| \delta_{4} \int_{0}^{\eta_{4}} \frac{\left(\eta_{4}-q s\right)^{(\beta-1)}}{\Gamma_{q}(\beta)} L_{2}(s) d_{q} s \\
& \text { and } r_{2}=M_{2} A_{2} /\left(1-\kappa_{2}\right) .+\left|\alpha_{4}\right| \delta_{4} \int_{0}^{1} \frac{(1-q s)^{(\beta-1)}}{\Gamma_{q}(\beta)} L_{2}(s) d_{q} s \\
&\left.+\left|\beta_{4}\right| \delta_{4} \int_{0}^{1} \frac{(1-q s)^{(\beta-2)}}{\Gamma_{q}(\beta-1)} L_{2}(s) d_{q} s\right\} \\
& M_{2} A_{2}+r_{2} \kappa_{2}
\end{aligned}
$$

\section{Therefore, we obtain}

$\|T(u, v)\|=\max \left\{\left(T_{1} v, T_{2} u\right)\right\}=\max \left\{\left\|T_{1} v\right\|,\left\|T_{2} u\right\|\right\}=r$.

From the last estimate we can choose $r=\max \left\{r_{1}, r_{2}\right\}$; then, for every $(u, v) \in U$, we have $T U \subset U$.

In order to show that $T$ is a contraction, let $u, v, u_{1}, v_{1} \in X$, and, for any $t \in[0,1]$, we get

$$
\begin{aligned}
& \left\|T_{1} v-T_{1} v_{1}\right\| \\
& \leq \sup _{t \in[0,1]}\left\{\int_{0}^{t} \frac{(t-q s)^{(\alpha-1)}}{\Gamma_{q}(\alpha)}\left|f(s, v(s))-f\left(s, v_{1}(s)\right)\right| d_{q} s\right. \\
& +\left|\frac{\gamma_{1}}{\Delta}\left[\left(\alpha_{2}-\gamma_{2}\right) t-\left(\alpha_{2}+\beta_{2}-\gamma_{2} \eta_{2}\right)\right]\right| \\
& \times \int_{0}^{\eta_{1}} \frac{\left(\eta_{1}-q s\right)^{(\alpha-1)}}{\Gamma_{q}(\alpha)}\left|f(s, v(s))-f\left(s, v_{1}(s)\right)\right| d_{q} s \\
& +\left|\frac{\gamma_{2}}{\Delta}\left[\left(\alpha_{1}-\gamma_{1}\right) t+\left(\beta_{1}+\gamma_{1} \eta_{1}\right)\right]\right| \\
& \times \int_{0}^{\eta_{2}} \frac{\left(\eta_{2}-q s\right)^{(\alpha-1)}}{\Gamma_{q}(\alpha)}\left|f(s, v(s))-f\left(s, v_{1}(s)\right)\right| d_{q} s \\
& +\left|\frac{\alpha_{2}}{\Delta}\left[\left(\alpha_{1}-\gamma_{1}\right) t+\left(\beta_{1}+\gamma_{1} \eta_{1}\right)\right]\right| \\
& \times \int_{0}^{1} \frac{(1-q s)^{(\alpha-1)}}{\Gamma_{q}(\alpha)}\left|f(s, v(s))-f\left(s, v_{1}(s)\right)\right| d_{q} s \\
& +\left|\frac{\beta_{2}}{\Delta}\left[\left(\alpha_{1}-\gamma_{1}\right) t+\left(\beta_{1}+\gamma_{1} \eta_{1}\right)\right]\right| \\
& \left.\times \int_{0}^{1} \frac{(1-q s)^{(\alpha-2)}}{\Gamma_{q}(\alpha-1)}\left|f(s, v(s))-f\left(s, v_{1}(s)\right)\right| d_{q} s\right\} \\
& \leq \sup _{t \in[0,1]}\left\{\int_{0}^{t} \frac{(t-q s)^{(\alpha-1)}}{\Gamma_{q}(\alpha)} L_{1}(s) d_{q} s\right. \\
& +\left|\frac{\gamma_{1}}{\Delta}\left[\left(\alpha_{2}-\gamma_{2}\right) t-\left(\alpha_{2}+\beta_{2}-\gamma_{2} \eta_{2}\right)\right]\right| \\
& \times \int_{0}^{\eta_{1}} \frac{\left(\eta_{1}-q s\right)^{(\alpha-1)}}{\Gamma_{q}(\alpha)} L_{1}(s) d_{q} s \\
& +\left|\frac{\gamma_{2}}{\Delta}\left[\left(\alpha_{1}-\gamma_{1}\right) t+\left(\beta_{1}+\gamma_{1} \eta_{1}\right)\right]\right| \\
& \times \int_{0}^{\eta_{2}} \frac{\left(\eta_{2}-q s\right)^{(\alpha-1)}}{\Gamma_{q}(\alpha)} L_{1}(s) d_{q} s \\
& +\left|\frac{\alpha_{2}}{\Delta}\left[\left(\alpha_{1}-\gamma_{1}\right) t+\left(\beta_{1}+\gamma_{1} \eta_{1}\right)\right]\right| \\
& \times \int_{0}^{1} \frac{(1-q s)^{(\alpha-1)}}{\Gamma_{q}(\alpha)} L_{1}(s) d_{q} s
\end{aligned}
$$




$$
\begin{aligned}
&+\left|\frac{\beta_{2}}{\Delta}\left[\left(\alpha_{1}-\gamma_{1}\right) t+\left(\beta_{1}+\gamma_{1} \eta_{1}\right)\right]\right| \\
&\left.\times \int_{0}^{1} \frac{(1-q s)^{(\alpha-2)}}{\Gamma_{q}(\alpha-1)} L_{1}(s) d_{q} s\right\}\left\|v-v_{1}\right\| \\
& \leq \sup _{t \in[0,1]}\left\{\int_{0}^{t} \frac{(t-q s)^{(\alpha-1)}}{\Gamma_{q}(\alpha)} L_{1}(s) d_{q} s\right. \\
&+\left|\gamma_{1}\right| \delta_{1} \int_{0}^{\eta_{1}} \frac{\left(\eta_{1}-q s\right)^{(\alpha-1)}}{\Gamma_{q}(\alpha)} L_{1}(s) d_{q} s \\
&+\left|\gamma_{2}\right| \delta_{2} \int_{0}^{\eta_{2}} \frac{\left(\eta_{2}-q s\right)^{(\alpha-1)}}{\Gamma_{q}(\alpha)} L_{1}(s) d_{q} s \\
&+\left|\alpha_{2}\right| \delta_{2} \int_{0}^{1} \frac{(1-q s)^{(\alpha-1)}}{\Gamma_{q}(\alpha)} L_{1}(s) d_{q} s \\
&\left.+\left|\beta_{2}\right| \delta_{2} \int_{0}^{1} \frac{(1-q s)^{(\alpha-2)}}{\Gamma_{q}(\alpha-1)} L_{1}(s) d_{q} s\right\}\left\|v-v_{1}\right\|,
\end{aligned}
$$

which, in view of $\kappa_{1}<1$ and (31), implies that

$$
\left\|T_{1} v(t)-T_{1} v_{1}(t)\right\| \leq \kappa_{1}\left\|v-v_{1}\right\| .
$$

Similarly, we have $\left\|T_{2} u-T_{2} u_{1}\right\| \leq \kappa_{2}\left\|u-u_{1}\right\|$.

Thus, we have

$$
\begin{aligned}
\| T & (u, v)-T\left(u_{1}, v_{1}\right) \| \\
& =\left\|\left(T_{1} v-T_{1} v_{1}, T_{2} u-T_{2} u_{1}\right)\right\| \\
& =\max \left\{\left\|T_{1} v-T_{1} v_{1}\right\|,\left\|T_{2} u-T_{2} u_{1}\right\|\right\} \\
& \leq \max \left\{\kappa_{1}, \kappa_{2}\right\} \max \left\{\left\|v-v_{1}\right\|,\left\|u-u_{1}\right\|\right\} \\
& =\max \left\{\kappa_{1}, \kappa_{2}\right\}\left\|\left(v-v_{1}, u-u_{1}\right)\right\| .
\end{aligned}
$$

Since $\kappa_{1}<1, \kappa_{2}<1$, therefore, the operator $T$ is a contraction. Hence, by Banach's contraction principle, the operator $T$ has a unique fixed point, which is the unique solution of the system (1). This completes the proof.

The second result is based on the nonlinear alternative of Leray-Schauder type (Lemma 9).

Theorem 12. Assume that $f, g:[0,1] \times \mathbb{R} \rightarrow \mathbb{R}$ are continuous functions and the following conditions hold:

(H2) there exist four functions $p_{i}(t), q_{i}(t) \in L^{1}\left([0,1], \mathbb{R}^{+}\right)$, $i=1,2$, and two nondecreasing functions $\varphi, \psi: \mathbb{R}^{+} \rightarrow$ $\mathbb{R}^{+}$, such that

$$
\begin{aligned}
& |f(t, x)| \leq p_{1}(t) \varphi(\|x\|)+q_{1}(t), \\
& |g(t, y)| \leq p_{2}(t) \psi(\|y\|)+q_{2}(t),
\end{aligned}
$$

where $(t, x),(t, y) \in[0,1] \times \mathbb{R}$.
(H3) There exists a constant $M>0$ such that

$$
\begin{aligned}
& \varphi(M) \omega_{1}+\omega_{2}<M, \\
& \psi(M) \omega_{1}+\varpi_{2}<M,
\end{aligned}
$$

where

$$
\begin{aligned}
\omega_{1}:= & \left(1+\left|\alpha_{2}\right| \delta_{2}\right)\left(I_{q}^{\alpha} p_{1}\right)(1)+\left|\gamma_{1}\right| \delta_{1}\left(I_{q}^{\alpha} p_{1}\right)\left(\eta_{1}\right) \\
& +\left|\gamma_{2}\right| \delta_{2}\left(I_{q}^{\alpha} p_{1}\right)\left(\eta_{2}\right)+\left|\beta_{2}\right| \delta_{2}\left(I_{q}^{\alpha-1} p_{1}\right)(1), \\
\omega_{2}:= & \left(1+\left|\alpha_{2}\right| \delta_{2}\right)\left(I_{q}^{\alpha} q_{1}\right)(1)+\left|\gamma_{1}\right| \delta_{1}\left(I_{q}^{\alpha} q_{1}\right)\left(\eta_{1}\right) \\
& +\left|\gamma_{2}\right| \delta_{2}\left(I_{q}^{\alpha} q_{1}\right)\left(\eta_{2}\right)+\left|\beta_{2}\right| \delta_{2}\left(I_{q}^{\alpha-1} q_{1}\right)(1), \\
\omega_{1}:= & \left(1+\left|\alpha_{4}\right| \delta_{4}\right)\left(I_{q}^{\beta} p_{2}\right)(1)+\left|\gamma_{3}\right| \delta_{3}\left(I_{q}^{\beta} p_{2}\right)\left(\eta_{3}\right) \\
& +\left|\gamma_{4}\right| \delta_{4}\left(I_{q}^{\beta} p_{2}\right)\left(\eta_{4}\right)+\left|\beta_{4}\right| \delta_{4}\left(I_{q}^{\beta-1} p_{2}\right)(1), \\
\omega_{2}:= & \left(1+\left|\alpha_{4}\right| \delta_{4}\right)\left(I_{q}^{\beta} q_{2}\right)(1)+\left|\gamma_{3}\right| \delta_{3}\left(I_{q}^{\beta} q_{2}\right)\left(\eta_{3}\right) \\
& +\left|\gamma_{4}\right| \delta_{4}\left(I_{q}^{\beta} q_{2}\right)\left(\eta_{4}\right)+\left|\beta_{4}\right| \delta_{4}\left(I_{q}^{\beta-1} q_{2}\right)(1) .
\end{aligned}
$$

Then system (1) has at least one solution on $[0,1]$.

Proof. Consider the operator $T: X \times X \rightarrow X \times X$ defined by (25). The proof consists of several steps. As a first step, it will be shown that $T$ maps bounded sets into bounded sets in $X \times X$. For a positive number $r$, let $U=\{(u, v) \in X \times X$ : $\|(u, v)\| \leq r\}$ be bounded set in $X \times X$; then, for $(u, v) \in U$, we have

$$
\begin{aligned}
\left\|T_{1} v\right\| & \leq \sup _{t \in[0,1]}\left\{\int_{0}^{t} \frac{(t-q s)^{(\alpha-1)}}{\Gamma_{q}(\alpha)}|f(s, v(s))| d_{q} s\right. \\
& +\left|\frac{\gamma_{1}}{\Delta}\left[\left(\alpha_{2}-\gamma_{2}\right) t-\left(\alpha_{2}+\beta_{2}-\gamma_{2} \eta_{2}\right)\right]\right| \\
& \times \int_{0}^{\eta_{1}} \frac{\left(\eta_{1}-q s\right)^{(\alpha-1)}}{\Gamma_{q}(\alpha)}|f(s, v(s))| d_{q} s \\
& +\left|\frac{\gamma_{2}}{\Delta}\left[\left(\alpha_{1}-\gamma_{1}\right) t+\left(\beta_{1}+\gamma_{1} \eta_{1}\right)\right]\right| \\
& \times \int_{0}^{\eta_{2}} \frac{\left(\eta_{2}-q s\right)^{(\alpha-1)}}{\Gamma_{q}(\alpha)}|f(s, v(s))| d_{q} s \\
& +\left|\frac{\alpha_{2}}{\Delta}\left[\left(\alpha_{1}-\gamma_{1}\right) t+\left(\beta_{1}+\gamma_{1} \eta_{1}\right)\right]\right| \\
& \times \int_{0}^{1} \frac{(1-q s)^{(\alpha-1)}}{\Gamma_{q}(\alpha)}|f(s, v(s))| d_{q} s \\
& +\left|\frac{\beta_{2}}{\Delta}\left[\left(\alpha_{1}-\gamma_{1}\right) t+\left(\beta_{1}+\gamma_{1} \eta_{1}\right)\right]\right| \\
& \left.\times \int_{0}^{1} \frac{(1-q s)^{(\alpha-2)}}{\Gamma_{q}(\alpha-1)}|f(s, v(s))| d_{q} s\right\}
\end{aligned}
$$




$$
\begin{aligned}
& \leq \sup _{t \in[0,1]}\left\{\int_{0}^{t} \frac{(t-q s)^{(\alpha-1)}}{\Gamma_{q}(\alpha)}\left[p_{1}(s) \varphi(\|v\|)+q_{1}(s)\right] d_{q} s\right. \\
& +\left|\gamma_{1}\right| \delta_{1} \int_{0}^{\eta_{1}} \frac{\left(\eta_{1}-q s\right)^{(\alpha-1)}}{\Gamma_{q}(\alpha)} \\
& \times\left[p_{1}(s) \varphi(\|v\|)+q_{1}(s)\right] d_{q} s \\
& +\left|\gamma_{2}\right| \delta_{2} \int_{0}^{\eta_{2}} \frac{\left(\eta_{2}-q s\right)^{(\alpha-1)}}{\Gamma_{q}(\alpha)} \\
& \times\left[p_{1}(s) \varphi(\|v\|)+q_{1}(s)\right] d_{q} s \\
& +\left|\alpha_{2}\right| \delta_{2} \int_{0}^{1} \frac{(1-q s)^{(\alpha-1)}}{\Gamma_{q}(\alpha)} \\
& \times\left[p_{1}(s) \varphi(\|v\|)+q_{1}(s)\right] d_{q} s \\
& +\left|\beta_{2}\right| \delta_{2} \int_{0}^{1} \frac{(1-q s)^{(\alpha-2)}}{\Gamma_{q}(\alpha-1)} \\
& \left.\times\left[p_{1}(s) \varphi(\|v\|)+q_{1}(s)\right] d_{q} s\right\} \\
& \leq \varphi(r)\left\{\int_{0}^{1} \frac{(1-q s)^{(\alpha-1)}}{\Gamma_{q}(\alpha)} p_{1}(s) d_{q} s\right. \\
& +\left|\gamma_{1}\right| \delta_{1} \int_{0}^{\eta_{1}} \frac{\left(\eta_{1}-q s\right)^{(\alpha-1)}}{\Gamma_{q}(\alpha)} p_{1}(s) d_{q} s \\
& +\left|\gamma_{2}\right| \delta_{2} \int_{0}^{\eta_{2}} \frac{\left(\eta_{2}-q s\right)^{(\alpha-1)}}{\Gamma_{q}(\alpha)} p_{1}(s) d_{q} s \\
& +\left|\alpha_{2}\right| \delta_{2} \int_{0}^{1} \frac{(1-q s)^{(\alpha-1)}}{\Gamma_{q}(\alpha)} p_{1}(s) d_{q} s \\
& \left.+\left|\beta_{2}\right| \delta_{2} \int_{0}^{1} \frac{(1-q s)^{(\alpha-2)}}{\Gamma_{q}(\alpha-1)} p_{1}(s) d_{q} s\right\} \\
& +\left\{\int_{0}^{1} \frac{(1-q s)^{(\alpha-1)}}{\Gamma_{q}(\alpha)} q_{1}(s) d_{q} s\right. \\
& +\left|\gamma_{1}\right| \delta_{1} \int_{0}^{\eta_{1}} \frac{\left(\eta_{1}-q s\right)^{(\alpha-1)}}{\Gamma_{q}(\alpha)} q_{1}(s) d_{q} s \\
& +\left|\gamma_{2}\right| \delta_{2} \int_{0}^{\eta_{2}} \frac{\left(\eta_{2}-q s\right)^{(\alpha-1)}}{\Gamma_{q}(\alpha)} q_{1}(s) d_{q} s \\
& +\left|\alpha_{2}\right| \delta_{2} \int_{0}^{1} \frac{(1-q s)^{(\alpha-1)}}{\Gamma_{q}(\alpha)} q_{1}(s) d_{q} s \\
& \left.+\left|\beta_{2}\right| \delta_{2} \int_{0}^{1} \frac{(1-q s)^{(\alpha-2)}}{\Gamma_{q}(\alpha-1)} q_{1}(s) d_{q} s\right\} \text {. }
\end{aligned}
$$

As before, it can be shown that

$$
\left\|T_{1} v\right\| \leq \varphi(r) \omega_{1}+\omega_{2}
$$

Similarly, we have

$$
\left\|T_{2} u\right\| \leq \psi(r) \omega_{1}+\omega_{2}
$$

Thus, $T$ maps bounded sets into bounded sets in $X \times X$.

Next, we show that $T$ maps bounded sets into equicontinuous sets of $X \times X$. Let $t_{1}, t_{2} \in[0,1]$ with $t_{1}<t_{2}$ and $(u, v) \in U$, where $U$ is a bounded set of $X \times X$. Then taking into consideration the inequality $\left(t_{2}-q s\right)^{(\alpha-1)}-\left(t_{1}-q s\right)^{(\alpha-1)} \leq$ $\left(t_{2}-t_{1}\right)$, for $0<t_{1}<t_{2}$, we obtain

$$
\begin{aligned}
& \left|\left(T_{1} v\right)\left(t_{2}\right)-\left(T_{1} v\right)\left(t_{1}\right)\right| \\
& \leq \mid \int_{0}^{t_{2}} \frac{\left(t_{2}-q s\right)^{(\alpha-1)}}{\Gamma_{q}(\alpha)} f(s, v(s)) d_{q} s \\
& -\int_{0}^{t_{1}} \frac{\left(t_{1}-q s\right)^{(\alpha-1)}}{\Gamma_{q}(\alpha)} f(s, v(s)) d_{q} s \\
& +\frac{1}{\Delta}\left\{\left|\gamma_{1}\left(\alpha_{2}-\gamma_{2}\right)\right|\left(t_{2}-t_{1}\right)\right. \\
& \times \int_{0}^{\eta_{1}} \frac{\left(\eta_{1}-q s\right)^{(\alpha-1)}}{\Gamma_{q}(\alpha)}|f(s, v(s))| d_{q} s \\
& +\gamma_{2}\left|\alpha_{1}-\gamma_{1}\right|\left(t_{2}-t_{1}\right) \\
& \times \int_{0}^{\eta_{2}} \frac{\left(\eta_{2}-q s\right)^{(\alpha-1)}}{\Gamma_{q}(\alpha)}|f(s, v(s))| d_{q} s \\
& +\left|\alpha_{2}\right|\left|\alpha_{1}-\gamma_{1}\right|\left(t_{2}-t_{1}\right) \\
& \times \int_{0}^{1} \frac{(1-q s)^{(\alpha-1)}}{\Gamma_{q}(\alpha)}|f(s, v(s))| d_{q} s \\
& +\left|\beta_{2}\right|\left|\alpha_{1}-\gamma_{1}\right|\left(t_{2}-t_{1}\right) \\
& \left.\times \int_{0}^{1} \frac{(1-q s)^{(\alpha-2)}}{\Gamma_{q}(\alpha-1)}|f(s, v(s))| d_{q} s\right\} \\
& \leq \mid \int_{0}^{t_{1}} \frac{\left[\left(t_{2}-q s\right)^{(\alpha-1)}-\left(t_{1}-q s\right)^{(\alpha-1)}\right]}{\Gamma_{q}(\alpha)} \\
& \times\left[p_{1}(s) \varphi(\|v\|)+q_{1}(s)\right] d_{q} s \\
& +\int_{t_{1}}^{t_{2}} \frac{\left(t_{2}-q s\right)^{(\alpha-1)}}{\Gamma_{q}(\alpha)}\left[p_{1}(s) \varphi(\|v\|)+q_{1}(s)\right] d_{q} s
\end{aligned}
$$




$$
\begin{aligned}
& +\frac{1}{\Delta}\left\{\left|\gamma_{1}\left(\alpha_{2}-\gamma_{2}\right)\right|\left(t_{2}-t_{1}\right)\right. \\
& \times \int_{0}^{\eta_{1}} \frac{\left(\eta_{1}-q s\right)^{(\alpha-1)}}{\Gamma_{q}(\alpha)}\left[p_{1}(s) \varphi(\|v\|)+q_{1}(s)\right] d_{q} s \\
& +\gamma_{2}\left|\alpha_{1}-\gamma_{1}\right|\left(t_{2}-t_{1}\right) \\
& \times \int_{0}^{\eta_{2}} \frac{\left(\eta_{2}-q s\right)^{(\alpha-1)}}{\Gamma_{q}(\alpha)}\left[p_{1}(s) \varphi(\|v\|)+q_{1}(s)\right] d_{q} s \\
& +\left|\alpha_{2}\right|\left|\alpha_{1}-\gamma_{1}\right|\left(t_{2}-t_{1}\right) \\
& \times \int_{0}^{1} \frac{(1-q s)^{(\alpha-1)}}{\Gamma_{q}(\alpha)}\left[p_{1}(s) \varphi(\|v\|)+q_{1}(s)\right] d_{q} s \\
& +\left|\beta_{2}\right|\left|\alpha_{1}-\gamma_{1}\right|\left(t_{2}-t_{1}\right) \\
& \left.\times \int_{0}^{1} \frac{(1-q s)^{(\alpha-2)}}{\Gamma_{q}(\alpha)}\left[p_{1}(s) \varphi(\|v\|)+q_{1}(s)\right] d_{q} s\right\} \\
& \leq \mid \int_{0}^{t_{1}} \frac{\left(t_{2}-t_{1}\right)}{\Gamma_{q}(\alpha)}\left[p_{1}(s) \varphi(r)+q_{1}(s)\right] d_{q} s \\
& +\int_{t_{1}}^{t_{2}} \frac{\left(t_{2}-q s\right)^{(\alpha-1)}}{\Gamma_{q}(\alpha)}\left[p_{1}(s) \varphi(r)+q_{1}(s)\right] d_{q} s \\
& +\frac{1}{\Delta}\left\{\left|\gamma_{1}\left(\alpha_{2}-\gamma_{2}\right)\right|\left(t_{2}-t_{1}\right)\right. \\
& \times \int_{0}^{\eta_{1}} \frac{\left(\eta_{1}-q s\right)^{(\alpha-1)}}{\Gamma_{q}(\alpha)}\left[p_{1}(s) \varphi(r)+q_{1}(s)\right] d_{q} s \\
& +\gamma_{2}\left|\alpha_{1}-\gamma_{1}\right|\left(t_{2}-t_{1}\right) \\
& \times \int_{0}^{\eta_{2}} \frac{\left(\eta_{2}-q s\right)^{(\alpha-1)}}{\Gamma_{q}(\alpha)}\left[p_{1}(s) \varphi(r)+q_{1}(s)\right] d_{q} s \\
& +\left|\alpha_{2}\right|\left|\alpha_{1}-\gamma_{1}\right|\left(t_{2}-t_{1}\right) \\
& \times \int_{0}^{1} \frac{(1-q s)^{(\alpha-1)}}{\Gamma_{q}(\alpha)}\left[p_{1}(s) \varphi(r)+q_{1}(s)\right] d_{q} s \\
& +\left|\beta_{2}\right|\left|\alpha_{1}-\gamma_{1}\right|\left(t_{2}-t_{1}\right) \\
& \left.\times \int_{0}^{1} \frac{(1-q s)^{(\alpha-2)}}{\Gamma_{q}(\alpha)}\left[p_{1}(s) \varphi(r)+q_{1}(s)\right] d_{q} s\right\} .
\end{aligned}
$$

Clearly, the right-hand side of the above inequality tends to zero independently of $v \in U$ as $t_{2} \rightarrow t_{1}$. Thus, it follows by the Arzelá-Ascoli theorem that $T_{1}$ is completely continuous. Similarly, $T_{2}$ is completely continuous. Therefore, $T: X \times$ $X \rightarrow X \times X$ is completely continuous.

Let us set $\Omega=\{(u, v) \in U:\|(u, v)\|<M\}$. Note that the operator $T: \bar{\Omega} \rightarrow X \times X$ is continuous and completely continuous. From the choice of $\Omega$, assume that there is $(u, v) \epsilon$ $\partial \Omega$ such that $(u, v)=\lambda T(u, v)$, for some $\lambda \in(0,1)$. By $(\mathrm{H} 3)$, we obtain

$$
\begin{aligned}
\|(u, v)\| & =\lambda\|T(u, v)\|<\|T(u, v)\| \\
& =\max \left\{\left\|T_{1} v\right\|,\left\|T_{2} u\right\|\right\}<M,
\end{aligned}
$$

which is a contradiction. In consequence, by the nonlinear alternative of Leray-Schauder type (Lemma 9), we deduce that $T$ has a fixed point $(u, v) \in \bar{\Omega}$ which is a solution of the system (1). The proof is complete.

In the sequel we present two examples which illustrate Theorems 11 and 12.

\section{Examples}

Example 1. Consider the following fractional $q$-difference nonlocal boundary value problem:

$$
\begin{aligned}
&{ }^{C} D_{q}^{3 / 2} u(t)=\frac{L_{1}}{2}\left(v+\tan ^{-1} v+\sin t\right), \quad 0 \leq t \leq 1, \\
&{ }^{C} D_{q}^{7 / 4} v(t)= \frac{L_{2}}{3}\left(u+\tan ^{-1} u+\sin t\right), \quad 0 \leq t \leq 1, \\
& u(0)-\frac{1}{2} D_{q} u(0)=u\left(\frac{1}{3}\right), \\
& \frac{1}{4} u(1)+\frac{3}{4} D_{q} u(1)=u\left(\frac{2}{3}\right), \\
& v(0)-\frac{1}{2} D_{q} v(0)=v\left(\frac{1}{3}\right), \\
& \frac{1}{4} v(1)+\frac{3}{4} D_{q} v(1)=v\left(\frac{2}{3}\right) .
\end{aligned}
$$

In this case, $\alpha=3 / 2, \beta=7 / 4, \alpha_{1}=1, \beta_{1}=1 / 2, \alpha_{2}=1 / 4$, $\beta_{2}=3 / 4, \alpha_{3}=1, \beta_{3}=1 / 2, \alpha_{4}=1 / 4, \beta_{4}=3 / 4, \eta_{1}=1 / 3$, $\eta_{2}=2 / 3, \eta_{3}=1 / 3, \eta_{4}=2 / 3, \gamma_{1}=1=\gamma_{2}, \gamma_{3}=1=\gamma_{4}$, and $L_{1}, L_{2}$ are constants to be fixed later on. Moreover, $\Delta=5 / 8$, $\boldsymbol{\Delta}=5 / 8, \delta_{1}=26 / 15, \delta_{2}=4 / 3, \delta_{3}=26 / 15$, and $\delta_{4}=4 / 3$. Consider

$$
\begin{array}{r}
f(t, v)=\frac{L_{1}}{2}\left(v+\tan ^{-1} v+\sin t\right), \\
g(t, u)=\frac{L_{2}}{3}\left(u+\tan ^{-1} u+\sin t\right), \\
\quad t \in[0,1], u, v \in C[0,1] .
\end{array}
$$

Clearly, we have

$$
\begin{gathered}
\left|f(t, v)-f\left(t, v_{1}\right)\right| \leq L_{1}\left|v-v_{1}\right|, \\
\left|g(t, u)-g\left(t, u_{1}\right)\right| \leq L_{2}\left|u-u_{1}\right|, \\
\kappa_{1}:=\frac{L_{1}}{\Gamma_{1 / 2}(3 / 2)}\left(1+\frac{2 \sqrt{2}(13+10 \sqrt{2}+15 \sqrt{3})}{15 \sqrt{3}(2 \sqrt{2}-1)}\right), \\
\kappa_{2}:=\frac{L_{2}}{\Gamma_{1 / 2}(7 / 4)}\left(1+\frac{2 \sqrt{2}(13+10 \sqrt{2}+15 \sqrt{3})}{15 \sqrt{3}(2 \sqrt{2}-1)}\right) .
\end{gathered}
$$


Choose

$$
\begin{aligned}
& L_{1}:=\left[\frac{1}{\Gamma_{1 / 2}(3 / 2)}\left(1+\frac{2 \sqrt{2}(13+10 \sqrt{2}+15 \sqrt{3})}{15 \sqrt{3}(2 \sqrt{2}-1)}\right)\right]^{-1}, \\
& L_{2}:=\left[\frac{1}{\Gamma_{1 / 2}(7 / 4)}\left(1+\frac{2 \sqrt{2}(13+10 \sqrt{2}+15 \sqrt{3})}{15 \sqrt{3}(2 \sqrt{2}-1)}\right)\right]^{-1} .
\end{aligned}
$$

Hence all the assumptions of Theorem 11 are satisfied. Therefore, by Theorem 11, the problem (50) has a unique solution.

Example 2. Consider the following fractional boundary value problem:

$$
\begin{gathered}
{ }^{C} D_{q}^{3 / 2} u(t)=\frac{1}{4} \cos t^{2} \sin \left(\frac{|v|}{2}\right)+\frac{e^{-x^{2}\left(t^{2}+1\right)}}{1+\left(t^{2}+1\right)}+\frac{1}{3}, \\
0 \leq t \leq 1, \\
{ }^{C} D_{q}^{3 / 2} v(t)=\frac{1}{16 \pi} \sin (2 \pi u(t))+\frac{|u(t)|}{2(1+|u(t)|)}+\frac{1}{2}, \\
u(0)-\frac{1}{2} D_{q} u(0)=u\left(\frac{1}{3}\right), \\
\frac{1}{4} u(1)+\frac{3}{4} D_{q} u(1)=u\left(\frac{2}{3}\right), \\
v(0)-\frac{1}{2} D_{q} v(0)=v\left(\frac{1}{3}\right), \\
\frac{1}{4} v(1)+\frac{3}{4} D_{q} v(1)=v\left(\frac{2}{3}\right) .
\end{gathered}
$$

In this case, $\alpha=3 / 2, \beta=3 / 2, \alpha_{1}=1, \beta_{1}=1 / 2, \alpha_{2}=1 / 4$, $\beta_{2}=3 / 4, \alpha_{3}=1, \beta_{3}=1 / 2, \alpha_{4}=1 / 4, \beta_{4}=3 / 4, \eta_{1}=1 / 3$, $\eta_{2}=2 / 3, \eta_{3}=1 / 3, \eta_{4}=2 / 3, \gamma_{1}=1=\gamma_{2}, \gamma_{3}=1=\gamma_{4}$, and $L_{1}, L_{2}$ are constants to be fixed later on. Moreover, $\Delta=5 / 8$, $\boldsymbol{\Delta}=5 / 8, \delta_{1}=26 / 15, \delta_{2}=4 / 3, \delta_{3}=26 / 15$, and $\delta_{4}=4 / 3$. Clearly

$$
\begin{aligned}
|f(t, v)| & =\left|\frac{1}{4} \cos t^{2} \sin \left(\frac{|v|}{2}\right)+\frac{e^{-x^{2}\left(t^{2}+1\right)}}{1+\left(t^{2}+1\right)}+\frac{1}{3}\right| \\
& \leq \frac{1}{8}|v|+1, \\
|g(t, u)| & =\left|\frac{1}{16 \pi} \sin (2 \pi u(t))+\frac{|u(t)|}{2(1+|u(t)|)}+\frac{1}{2}\right| \\
& \leq \frac{1}{8}|u|+1 .
\end{aligned}
$$

Clearly $p_{1}=1 / 8, q_{1}=1, \varphi(M)=M, p_{2}=1 / 8$, $q_{2}=1$, and $\psi(M)=M$. Consequently, $\omega_{1} \approx 0.567129414$, $\omega_{2} \approx 4.536963312, \omega_{1} \approx 0.567129414, \omega_{2} \approx 4.536963312$, and conditions (43) imply that $M>10.48055997$. Thus, all the assumptions of Theorem 12 are satisfied. Therefore, the conclusion of Theorem 12 applies to problems (54).

\section{Conflict of Interests}

The authors declare that there is no conflict of interests regarding the publication of this paper.

\section{Acknowledgments}

This research was supported by the National Natural Science Foundation of China (11161027, 11262009, and 11226132), the Scientific Research Projects in Colleges and Universities of Gansu Province of China (2013A-043), the Fundamental Research Funds for the Gansu Universities (213061), the Fundamental Research Funds for the Gansu Universities (212084), the Youth Science Foundation of Lanzhou Jiaotong University (2012019), and the National Natural Science Foundation of China (11226132). The authors are thankful to the referees for their careful reading of the paper and insightful comments.

\section{References}

[1] A. A. Kilbas, H. M. Srivastava, and J. J. Trujillo, Theory and Applications of Fractional Differential Equations, vol. 204 of North-Holland Mathematics Studies, Elsevier Science, Amsterdam, The Netherlands, 2006.

[2] K. S. Miller and B. Ross, An Introduction to the Fractional Calculus and Fractional Differential Equations, John Wiley \& Sons, New York, NY, USA, 1993.

[3] K. B. Oldham and J. Spanier, The Fractional Calculus, Academic Press, London, UK, 1974.

[4] I. Podlubny, Fractional Differential Equations, vol. 198 of Mathematics in Science and Engineering, Academic Press, San Diego, Calif, USA, 1999.

[5] S. G. Samko, A. A. Kilbas, and O. I. Marichev, Fractional Integrals and Derivatives, Theory and Applications, Gordon and Breach Science, Yverdon, Switzerland, 1993.

[6] V. Lakshmikantham and A. S. Vatsala, "Basic theory of fractional differential equations," Nonlinear Analysis, vol. 69, no. 8, pp. 2677-2682, 2008.

[7] V. Lakshmikantham and A. S. Vatsala, "General uniqueness and monotone iterative technique for fractional differential equations," Applied Mathematics Letters, vol. 21, no. 8, pp. 828834, 2008.

[8] V. Lakshmikantham, "Theory of fractional functional differential equations," Nonlinear Analysis, vol. 69, no. 10, pp. 3337-3343, 2008.

[9] A. Babakhani and V. Daftardar-Gejji, "Existence of positive solutions of nonlinear fractional differential equations," Journal of Mathematical Analysis and Applications, vol. 278, no. 2, pp. 434-442, 2003.

[10] A. Babakhani and V. Daftardar-Gejji, "Existence of positive solutions for $N$-term non-autonomous fractional differential equations," Positivity, vol. 9, no. 2, pp. 193-206, 2005.

[11] A. Babakhani and V. Daftardar-Gejji, "Existence of positive solutions for multi-term non-autonomous fractional differential equations with polynomial coefficients," Abstract and Applied Analysis, vol. 2009, Article ID 768920, 12 pages, 2009.

[12] C. Bai, "Positive solutions for nonlinear fractional differential equations with coefficient that changes sign," Nonlinear Analysis, vol. 64, no. 4, pp. 677-685, 2006. 
[13] R. P. Agarwal, M. Benchohra, and S. Hamani, "Boundary value problems for differential inclusions with fractional order," Advanced Studies in Contemporary Mathematics, vol. 16, no. 2, pp. 181-196, 2008.

[14] W. Zhou and Y. Chu, "Existence of solutions for fractional differential equations with multi-point boundary conditions," Communications in Nonlinear Science and Numerical Simulation, vol. 17, no. 3, pp. 1142-1148, 2012.

[15] W. Zhou, J. Peng, and Y. Chu, "Multiple positive solutions for nonlinear semipositone fractional differential equations," Discrete Dynamics in Nature and Society, vol. 2012, Article ID 850871, 10 pages, 2012.

[16] W. Zhou and H. Liu, "Existence of weak solutions for nonlinear fractional differential inclusion with nonseparated boundary conditions," Journal of Applied Mathematics, vol. 2012, Article ID 530624, 13 pages, 2012.

[17] W. Zhou, Y. Chu, and D. Baleanu, "Uniqueness and existence of positive solutions for a multi-point boundary value problem of singular fractional differential equations," Advances in Diff erence Equations, vol. 2013, no. 114, 2013.

[18] W. Zhou and H. Liu, "Existence solutions for boundary value problem of nonlinear fractional $q$-difference equations," Advances in Difference Equations, vol. 2013, article 113, 12 pages, 2013.

[19] B. Ahmad and J. J. Nieto, "Existence results for a coupled system of nonlinear fractional differential equations with threepoint boundary conditions," Computers \& Mathematics with Applications, vol. 58, no. 9, pp. 1838-1843, 2009.

[20] X. Su, "Boundary value problem for a coupled system of nonlinear fractional differential equations," Applied Mathematics Letters, vol. 22, no. 1, pp. 64-69, 2009.

[21] J. Sun, Y. Liu, and G. Liu, "Existence of solutions for fractional differential systems with antiperiodic boundary conditions," Computers \& Mathematics with Applications, vol. 64, no. 6, pp. 1557-1566, 2012.

[22] J. Wang, H. Xiang, and Z. Liu, "Positive solution to nonzero boundary values problem for a coupled system of nonlinear fractional differential equations," International Journal of Differential Equations, vol. 2010, Article ID 186928, 12 pages, 2010.

[23] F. H. Jackson, "q-difference equations," American Journal of Mathematics, vol. 32, no. 4, pp. 305-314, 1910.

[24] F. H. Jackson, "On $q$-functions and a certain difference operator," Transactions of the Royal Society of Edinburgh, vol. 46, no. 2, pp. 253-281, 1909.

[25] T. Ernst, "A new notation for $q$-calculus and a new $q$-Taylor formula,” U.U.D.M. Report 1999: 25, Department of Mathematics, Up-psala University, 1999.

[26] T. Ernst, "The history of qi calculus and a new method," UUDM Report 2000, Department of Mathematics, Uppsala University, Uppsala, Sweden, 2000.

[27] B. Ahmad, "Boundary-value problems for nonlinear thirdorder q-difference equations," Electronic Journal of Differential Equations, vol. 94, pp. 1-7, 2011.

[28] B. Ahmad and S. K. Ntouyas, "Boundary value problems for q-difference inclusions," Abstract and Applied Analysis, Art. ID 292860, 15 pages, 2011.

[29] B. Ahmad, A. Alsaedi, and S. K. Ntouyas, "A study of second-order $q$-difference equations with boundary conditions," Advances in Difference Equations, vol. 2012, article 35, 2012.

[30] F. M. Atici and P. W. Eloe, "A transform method in discrete fractional calculus," International Journal of Difference Equations, vol. 2, no. 2, pp. 165-176, 2007.
[31] C. S. Goodrich, "Solutions to a discrete right-focal fractional boundary value problem," International Journal of Difference Equations, vol. 5, no. 2, pp. 195-216, 2010.

[32] C. S. Goodrich, "Continuity of solutions to discrete fractional initial value problems," Computers \& Mathematics with Applications, vol. 59, no. 11, pp. 3489-3499, 2010.

[33] F. M. Atici and P. W. Eloe, "Linear systems of fractional nabla difference equations," The Rocky Mountain Journal of Mathematics, vol. 41, no. 2, pp. 353-370, 2011.

[34] F. M. Atıcı and P. W. Eloe, "Two-point boundary value problems for finite fractional difference equations," Journal of Difference Equations and Applications, vol. 17, no. 4, pp. 445-456, 2011.

[35] N. R. O. Bastos, R. A. C. Ferreira, and D. F. M. Torres, "Discretetime fractional variational problems," Signal Processing, vol. 91, no. 3, pp. 513-524, 2011.

[36] N. R. O. Bastos, D. Mozyrska, and D. F. M. Torres, "Fractional derivatives and integrals on time scales via the inverse generalized Laplace transform," International Journal of Mathematics and Computation, vol. 11, no. J11, pp. 1-9, 2011.

[37] C. S. Goodrich, "Existence of a positive solution to a system of discrete fractional boundary value problems," Applied Mathematics and Computation, vol. 217, no. 9, pp. 4740-4753, 2011.

[38] C. S. Goodrich, "On discrete sequential fractional boundary value problems," Journal of Mathematical Analysis and Applications, vol. 385, no. 1, pp. 111-124, 2012.

[39] W. A. Al-Salam, "Some fractional \$q \$-integrals and \$q\$derivatives," Proceedings of the Edinburgh Mathematical Society II, vol. 15, no. 2, pp. 135-140, 1967.

[40] R. Agarwal, "Certain fractional $q$-integrals and $q$-derivatives," Proceedings of the Cambridge Philosophical Society, vol. 66, no. 2, pp. 365-370, 1969.

[41] M. El-Shahed and F. M. Al-Askar, "Positive solutions for boundary value problem of nonlinear fractional $q$-difference equation," ISRN Mathematical Analysis, vol. 2011, Article ID 385459, 12 pages, 2011.

[42] R. A. C. Ferreira, "Positive solutions for a class of boundary value problems with fractional $q$-differences," Computers \& Mathematics with Applications, vol. 61, no. 2, pp. 367-373, 2011.

[43] R. A. C. Ferreira, "Nontrivial solutions for fractional $q$ difference boundary value problems," Electronic Journal of Qualitative Theory of Differential Equations, vol. 70, 10 pages, 2010.

[44] J. R. Graef and L. Kong, "Positive solutions for a class of higher order boundary value problems with fractional q-derivatives," Applied Mathematics and Computation, vol. 218, no. 19, pp. 9682-9689, 2012.

[45] J. Ma and J. Yang, "Existence of solutions for multi-point boundary value problem of fractional q-difference equation," Electronic Journal of Qualitative Theory of Differential Equations, No. 92, 10 pages, 2011.

[46] G. Gasper and M. Rahman, Basic Hypergeometric Series, vol. 35 of Encyclopedia of Mathematics and its Applications, Cambridge University Press, Cambridge, UK, 1990.

[47] V. Kac and P. Cheung, Quantum Calculus, Springer, New York, NY, USA, 2002.

[48] P. M. Rajkovic, S. D. Marinkovic, and M. S. Stankovic, "On $q$-analogues of Caputo derivative and Mittag-Leffler function," Fractional Calculus \& Applied Analysis, vol. 10, no. 4, pp. 359373, 2007.

[49] A. Granas and J. Dugundji, Fixed Point Theory, Springer, New York, NY, USA, 2005. 


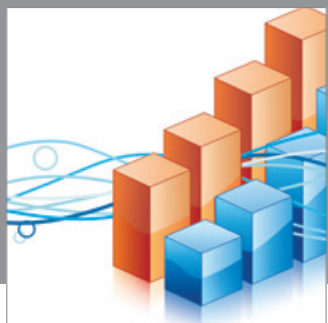

Advances in

Operations Research

mansans

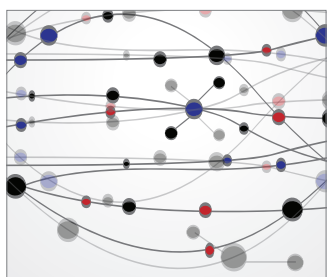

The Scientific World Journal
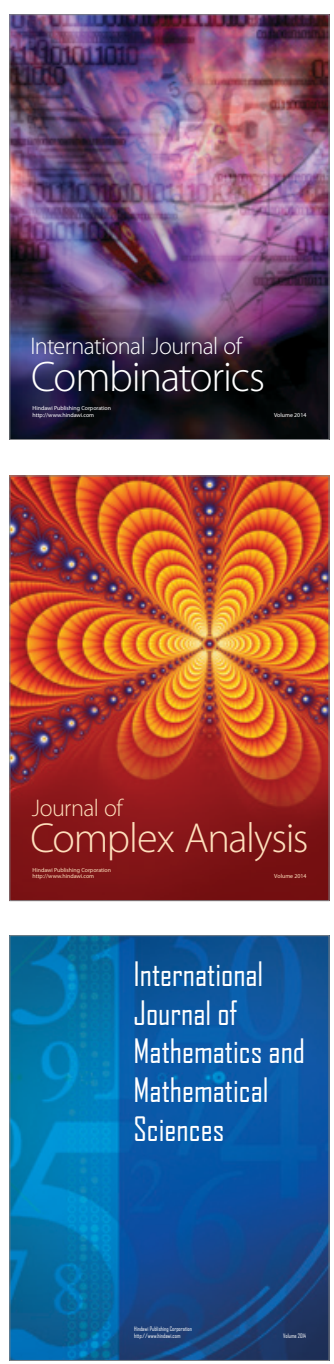
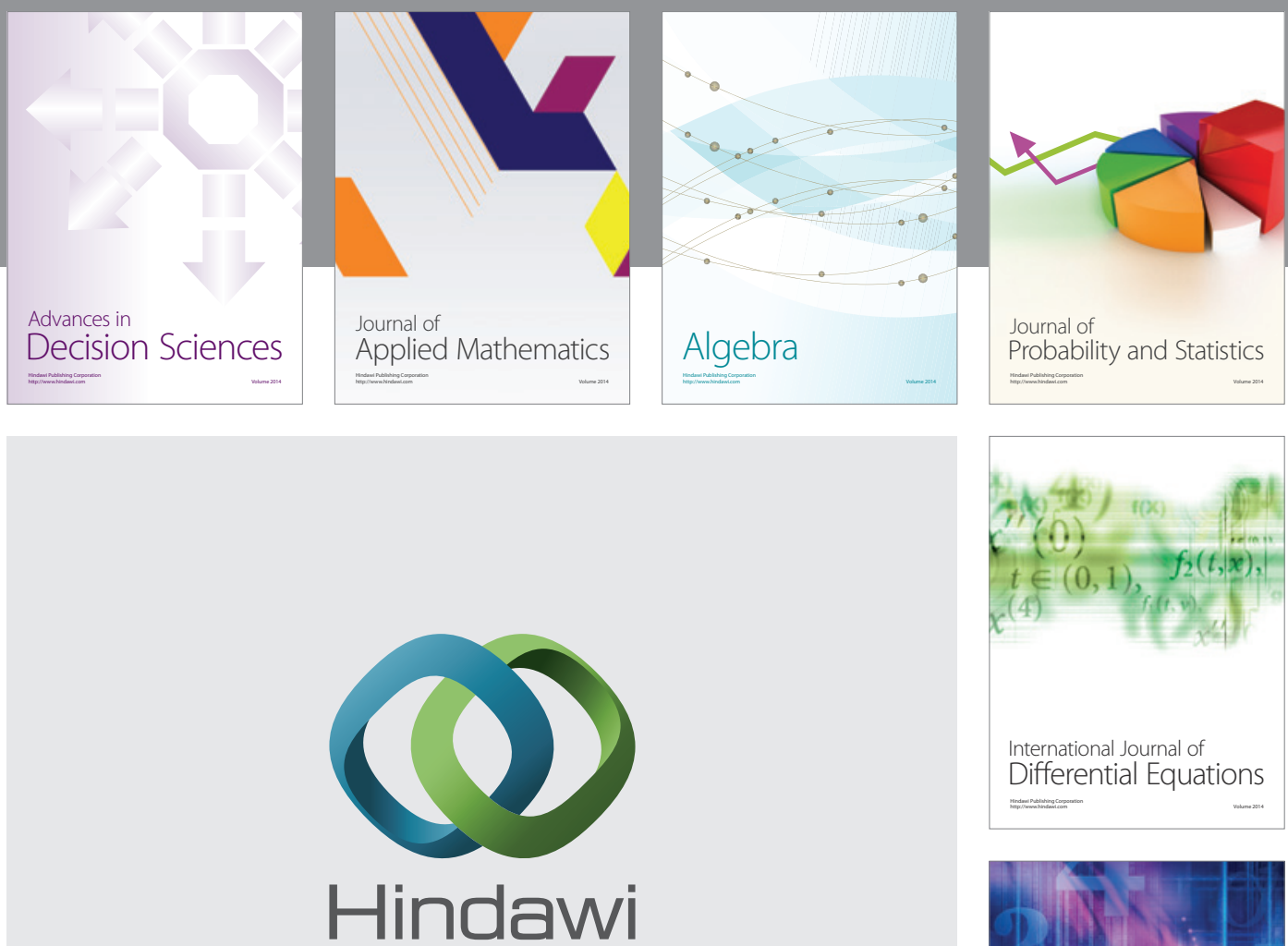

Submit your manuscripts at http://www.hindawi.com
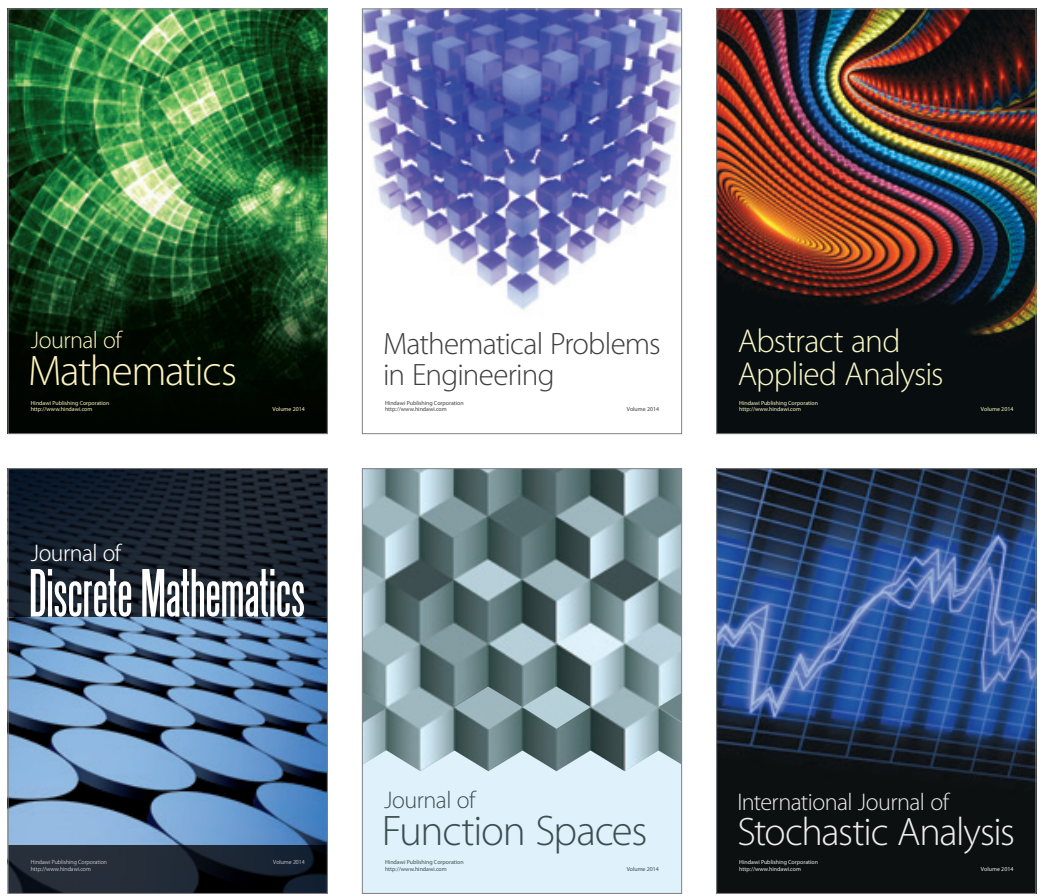

Journal of

Function Spaces

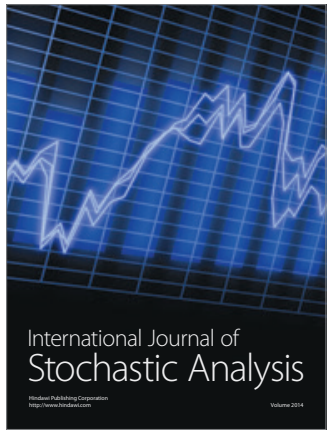

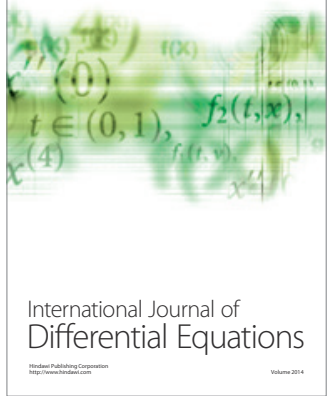
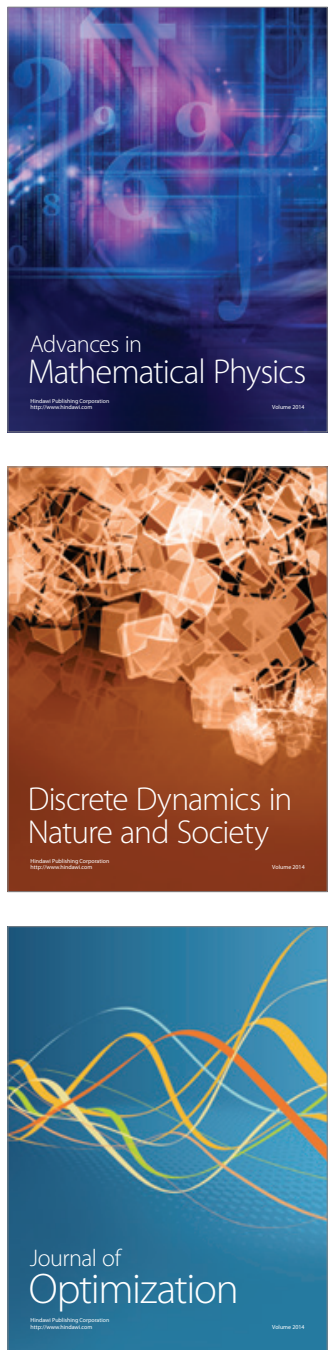